\title{
Lowered dose full-spine radiography in pediatric patients with idiopathic scoliosis
}

\author{
Caroline Ernst $^{1}{ }^{1} \cdot$ Nico Buls $^{1} \cdot$ Armand Laumen $^{1} \cdot$ Gert Van Gompel $^{1} \cdot$ Filip Verhelle $^{1} \cdot$ Johan de Mey $^{1}$
}

Received: 5 September 2017 / Revised: 8 March 2018 / Accepted: 22 March 2018 / Published online: 27 March 2018

(c) The Author(s) 2018

\begin{abstract}
Purpose To optimize our standard full-spine radiography with respect to diagnostic quality and dose.

Methods A phantom study was performed to establish an optimal posterior-anterior view (PA) full spine protocol having the lowest dose with non-inferior quality compared to standard. We then applied this protocol in 40 pediatric patients (group B). The radiographs were scored on six criteria by a pediatric radiologist and orthopedist and compared to the scores of 40 PA full spine radiographs performed in 2013 with standard protocol (group A). Radiation dose was assessed by dose area product (DAP) and effective dose (E). Statistical analysis included independent samples $t$ test, Mann-Whitney $U$ test and intra-class correlation coefficient (ICC).

Results An optimized protocol was defined $(0.2 \mathrm{~mm} \mathrm{Cu}$ filter, 0.87 relative exposure, with grid). Mean age was $13.3 \pm 1.6$ years for group A and $13.4 \pm 1.7$ years for group B. For group B, the mean DAP was $47.0 \mu \mathrm{Gy} \mathrm{m}^{2}$ with an E of $0.13 \mathrm{mSv}$. For group A, the mean DAP was $85.3 \mu \mathrm{Gy} \mathrm{m} \mathrm{m}^{2}$ with an $\mathrm{E}$ of $0.24 \mathrm{mSv}$. This represents a dose reduction of $45 \%$. Mean image quality scores for group A $(27.9 \pm 2.4)$ and group B $(28.1 \pm 2.3)$ were similar $(p=0.612)$. Interobserver agreement was observed to be excellent (ICC 0.92).

Conclusion This study demonstrates that a low-dose full-spine radiograph can be performed in patients with idiopathic scoliosis without loss of image quality.
\end{abstract}

Graphical abstract These slides can be retrieved under Electronic Supplementary Material.
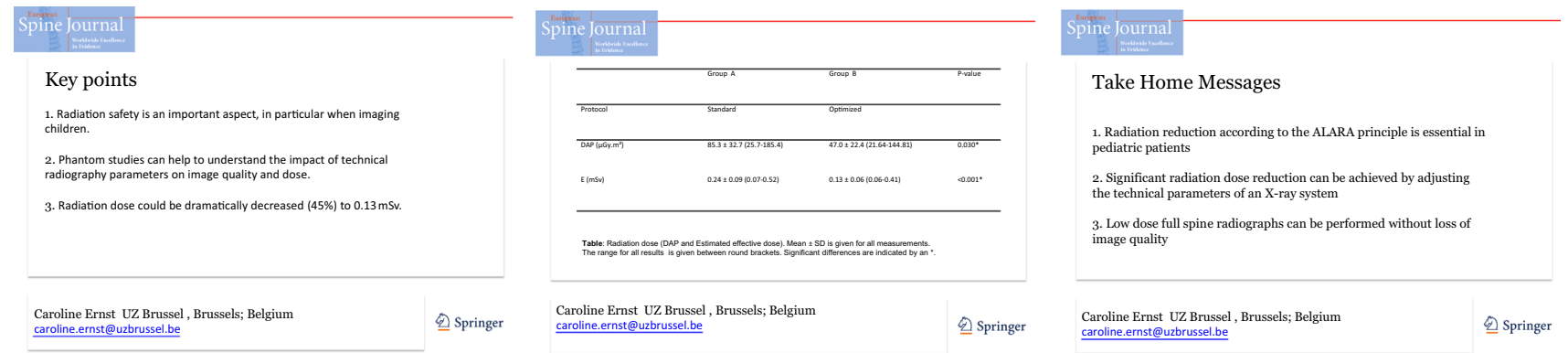

Keywords Idiopathic scoliosis $\cdot$ Full-spine radiography $\cdot$ Dose optimization $\cdot$ Adolescent $\cdot$ Image quality assessment

Electronic supplementary material The online version of this article (https://doi.org/10.1007/s00586-018-5561-9) contains supplementary material, which is available to authorized users.

Caroline Ernst

caroline.ernst@uzbrussel.be

1 UZ Brussel, Laarbeeklaan 101, 1090 Brussels, Belgium

\section{Introduction}

Idiopathic scoliosis is a three-dimensional spinal deformity which has a reported incidence of 2-4\% in a pediatric population aged six to fourteen [1]. Even though the initial diagnosis of scoliosis is usually made by physical examination, the definitive diagnosis and management is made 
by standing posteroanterior (PA) and lateral (LAT) radiographs of the full spine [2-4]. This imaging is needed to determine the type of curve and his severity to identify the presence of underlying pathologies, to decide whether treatment is necessary and if necessary repeated studies are required throughout the whole course $[2,4,5]$. Owing to the increased radiation sensitivity of children, numerous followup images and increased life expectancy, the chance of a radiation-induced abnormality is of concern $[4,6,7]$. The purpose of this study was to optimize our standard full-spine radiography protocol with respect to diagnostic quality and dose. Two studies were conducted to investigate this issue. First, a phantom study was performed where the impact of several technical parameters on image quality and radiation dose was evaluated and the optimal protocol was identified. In the prospective study, we performed this optimized (PA) full-spine radiograph in pediatric patients with idiopathic scoliosis and we compared radiation dose and image quality of these radiographs with earlier studies from our radiology archive.

\section{Materials and methods}

\section{Phantom study}

A phantom study was performed to assess the impact of technical parameters and to identify the optimal technique for the prospective study. Full-spine radiographs of a humanoid Alderson Rando phantom (The Phantom RANDO $^{\circledR}$, Alderson Research Laboratories Inc., Stamford, CT, USA) (Fig. 1) [8] were acquired with an Axiom Luminos dRF system (Siemens, Medical Solutions, Forchheim, Germany). The system is equipped with an automatic exposure control device (regulated by the relative exposure setting); the acquisition settings ( $\mathrm{kV}$, dose/pulse, filtration) were adapted in service mode.

The impact of following acquisition parameters was assessed: tube potential $(84.8$ or $101.9 \mathrm{kV})$, relative exposure setting $(0.87,1.09$ or 1.39 in arbitrary units), additional filtration (with or without $0.2 \mathrm{~mm} \mathrm{Cu}$ filter) and anti-scatter grid (yes or no). A total of 11 PA view protocols were investigated. The radiographs were scored on six image quality criteria on a five-point scale, as described in Table 1, (minimal 1 and maximal 5 points per criterion with a total score of minimal 5 and a maximal 30 points) by a pediatric radiologist (17 years of experience) and a pediatric orthopedist [9] years of experience), who were blinded to patient identity and clinical information. The images were evaluated on a PACS system (Impax 6.4.0, Agfa $^{\circledR}$ HealthCare, Mortsel, Belgium) on a 3 megapixel viewing station. Dose-related data were not displayed for this reading. The optimal protocol (protocol B) was identified as having the lowest DAP with non-inferior image quality compared to our standard protocol (protocol A: $84.8 \mathrm{kVp}$, with grid, 1.39 relative exposure, no filter).
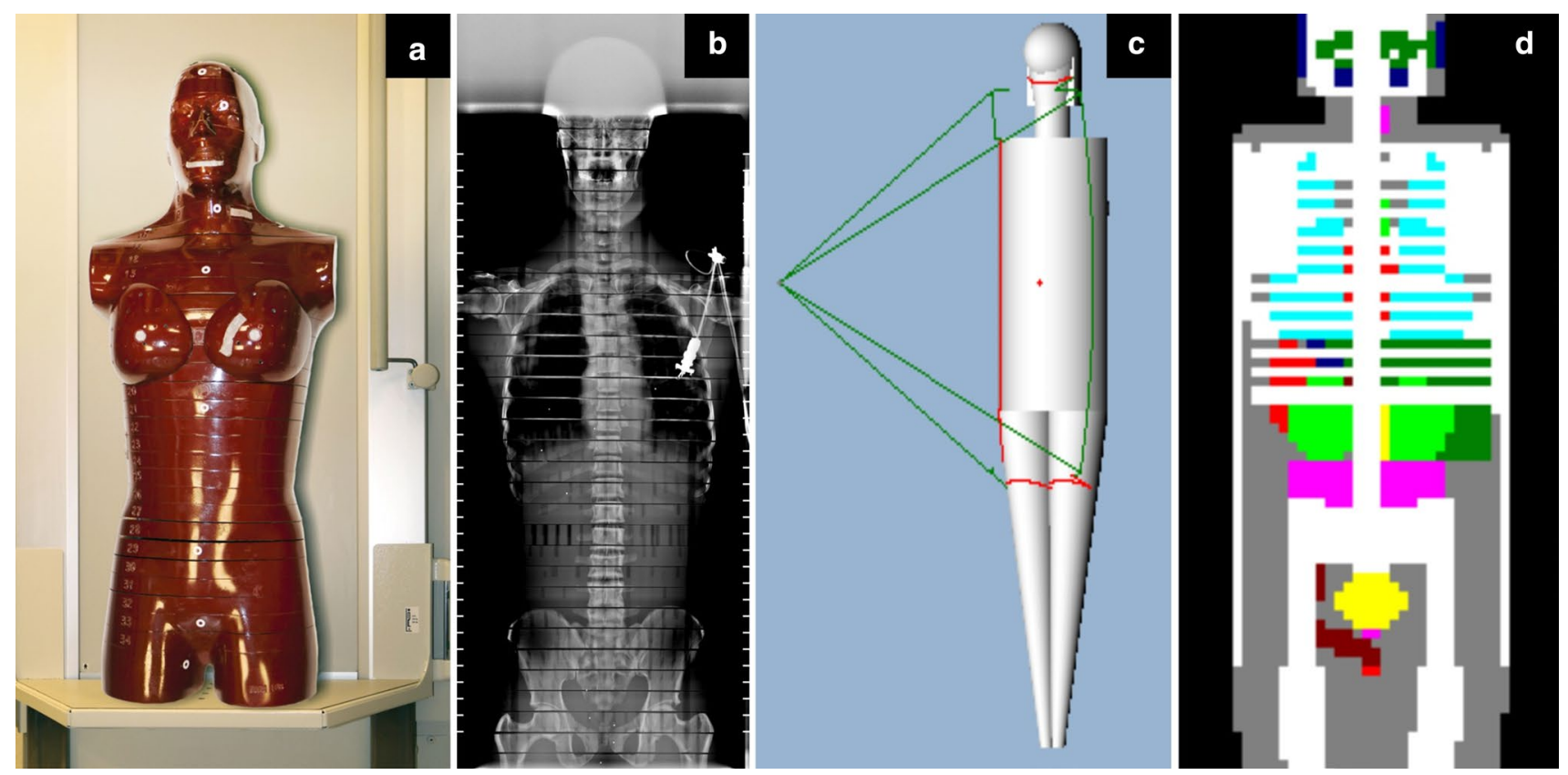

Fig. 1 a Rando Alderson phantom, $\mathbf{b}$ radiographic image of phantom, $\mathbf{c}$ exposure geometry of dosimetry model, $\mathbf{d}$ exposed organs and tissues in dosimetry model 
Table 1 Scoring list of subjective image quality

\begin{tabular}{lll}
\hline Score & $\begin{array}{l}\text { Bone sharpness; visibility of processus spinosus; delignation of the } \\
\text { intervertebral spaces }\end{array}$ & $\begin{array}{l}\text { Bone/soft tissue contrast; assessment of the } \\
\text { spinal curve; assessment of the Risser grade }\end{array}$ \\
\hline 1 & Structures cannot be identified & Unacceptable and nondiagnostic \\
2 & Although structures can be visualized, contours are blurred & Suboptimal, but still diagnostic \\
3 & Structures can be seen, but contours are barely sharp enough & Good \\
4 & Contours are not fully sharp, but structures are defined & Very good \\
5 & Structures are well defined with sharp contours & Excellent \\
\hline
\end{tabular}

Table 2 Patient characteristics: age and thoracic diameters

\begin{tabular}{llll}
\hline & Group A & Group B & $P$ value \\
\hline Protocol & Standard & Optimized & \\
Number of patients & 40 & 40 & \\
Age (years) & $13.3 \pm 1.6(10-16)$ & $13.4 \pm 1.7(10-16)$ & 0.426 \\
Craniocaudal diameter & $20.0 \pm 2.0(15.3-24.3)$ & $20.7 \pm 2.1(16.1-24.9)$ & 0.643 \\
Laterolateral diameter & $21.9 \pm 2.2(17.8-26.3)$ & $21.9 \pm 2.0(16.7-25.9)$ & 0.310 \\
\hline
\end{tabular}

Mean \pm SD is given for all measurements. The range for all results is given between round brackets

\section{Patient study}

This prospective study was approved by the local ethics committee (B.U.N. 143201422142). Written informed consent for participation was obtained from the parents or relatives of each patient. The optimized full-spine protocol (protocol B $84.8 \mathrm{kVp}$, with grid, 0.87 relative exposure, $0.2 \mathrm{~mm} \mathrm{Cu}$ filter), as identified from the phantom study, was performed in 40 consecutive patients with idiopathic scoliosis referred to our radiology department for a PA whole-spine radiograph between April 2015 and November 2015. Patients were excluded if they were not able to hold a standing position or if an in-brace full-spine radiograph was requested. Patient characteristics such as patient age and thoracic diameters are listed in Table 2. Images were acquired and scored with the same X-ray system and the same method as described in the phantom study. Patient sex, age, milliampere-second (mAs) and DAP were registered for all exams. The effective radiation doses were determined based on the Monte Carlo calculation using X-ray dosimetry software (PCXMC, version 2.0; Stuk, Helsinki, Finland) and based on the recorded DAP, patient size, and technical and geometric exposure parameters (Fig. 1c, d). Image quality and dosimetry were compared to data from archived images of 40 PA full spine $\mathrm{X}$-ray's performed with our standard protocol between April 2013 and November 2013 ( $85 \mathrm{kVp}, 1.39$ relative exposure, with grid, no filter) in a comparable patient group (consecutive patients selected within the same age limits [10-16]), acquired with the same X-ray system. The craniocaudal and laterolateral thoracic diameter was measured for each patient to compare patient size between both groups. Both groups

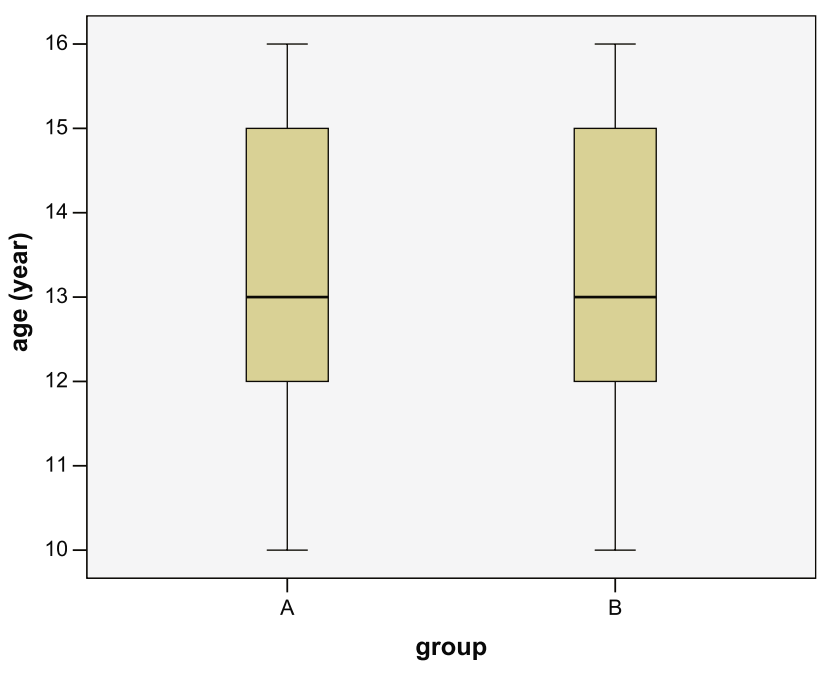

Fig. 2 Age of patient groups A and B

were similar with regard to age ( $p=0.426)$ (Fig. 2) and size, assessed by the craniocaudal $(p=0.643)$ and laterolateral thoracic diameter $(p=0.310)$. For objective image quality, the signal-to noise ratio (SNR) was considered. The SNR is a commonly used metric in dose optimization studies [9] and is expressed by the ratio of the signal (grey value) and the noise by its standard deviation. Circular regions of interest (ROIs) with constant area $\left(36.8 \pm 0.4 \mathrm{~mm}^{2}\right)$ were placed on the vertebral body of the eighth thoracic vertebra and in the soft tissue adjacent to this vertebra to calculate SNR in these structures. Subjective image quality was performed as described for the phantom study (Table 1). 


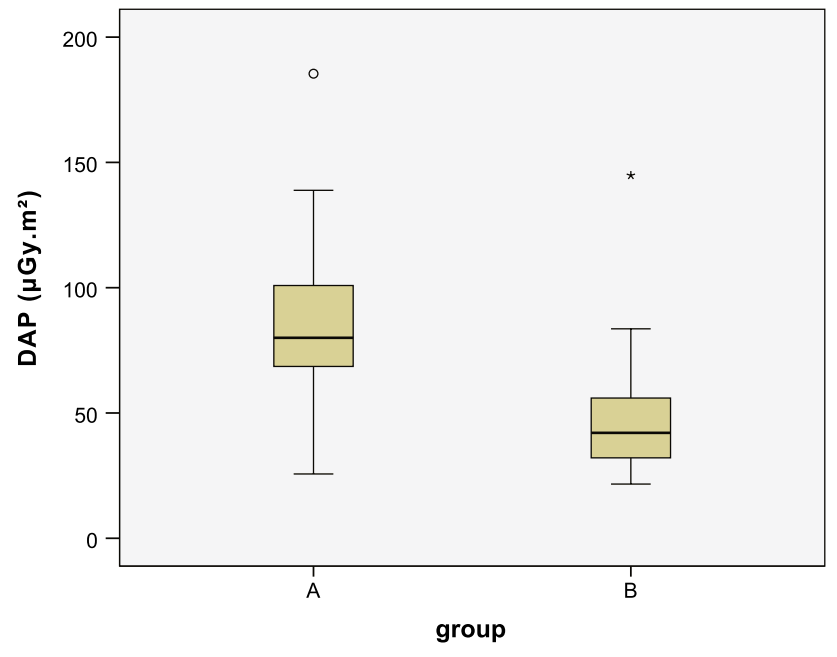

Fig. 3 Radiation dose (DAP) of patient groups A and B. DAP values of group $\mathrm{B}$ were observed to be lower than group A

Statistical analysis was performed using SPSS (IBM, v23). Independent samples t test was performed to test for inter-group differences in patient age and size (diameter), image quality (SNR) and radiation dose (E, DAP). Mann-Whitney $U$ test was performed to test for differences in subjective image quality. For all tests, a $p$ value of $<0.05$ was considered to represent a significant result. Interreader agreement was assessed by the intraclass correlation coefficient (ICC), with following levels of agreement: $<0$ poor; 0.0-0.20 slight; $0.21-0.40$ fair; $0.41-0.60$ moderate; 0.61-0.80 substantial; 0.81-1 almost perfect (Fig. 3).

\section{Results}

\section{Phantom study}

The acquisition parameters DAP and scores are displayed/ listed in Table 3. Protocol 1 represents our standard proto$\mathrm{col}=$ protocol A. The DAP for this protocol in our phantom study was of $194.73 \mu \mathrm{Gy} \mathrm{m}^{2}$ and the total mean score was 30. Protocol 8 is the protocol with the lowest DAP $\left(44.7 \mu \mathrm{Gy} \mathrm{m}^{2}\right)$ which still yielded the same quality as our standard protocol $($ total mean score $=30)=$ protocol B.

\section{Patient study}

The results for DAP and $\mathrm{E}$ for the optimized protocol (protocol B) and the standard protocol (protocol A) are listed in Table 4, the results for SNR and total mean score for both protocols are listed in Table 5.

The mean DAP was reduced from $85.3 \pm 32.7$ to $47.0 \pm 22.4 \mu \mathrm{Gy} \mathrm{m} \mathrm{m}^{2}$ and the mean $\mathrm{E}$ from $0.24 \pm 0.09$ to $0.13 \pm 0.06 \mathrm{mSv}$ (Table 4).

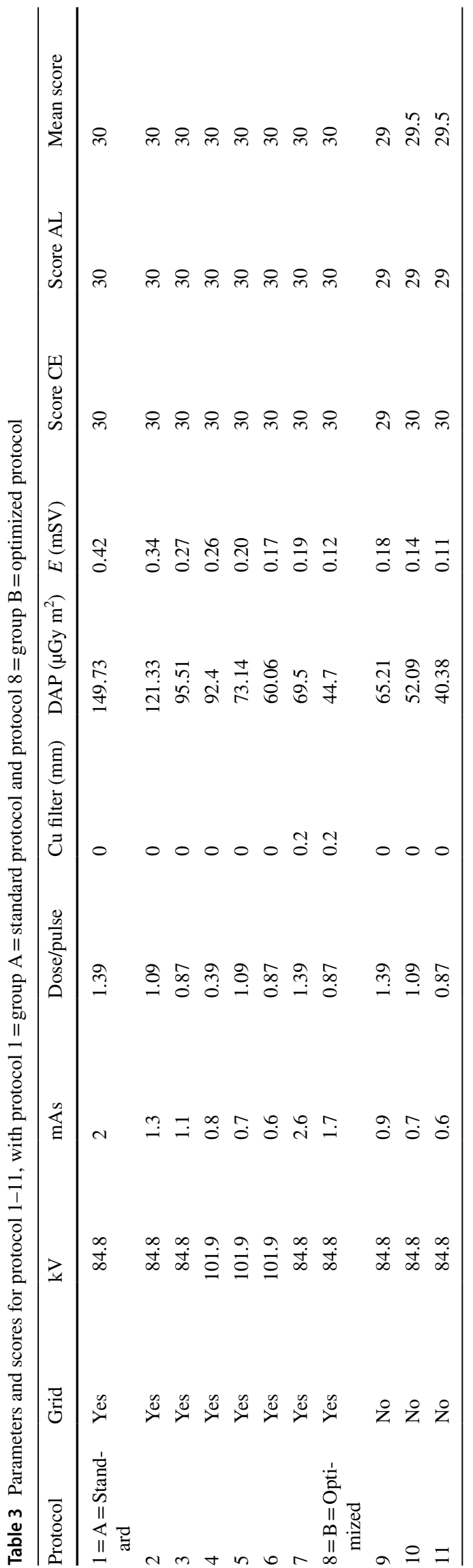


Table 4 Radiation dose (DAP and estimated effective dose)

\begin{tabular}{lllr}
\hline & Group A & Group B & $P$ value \\
\hline Protocol & Standard & Optimized & \\
DAP $\left(\mu \mathrm{Gy} \mathrm{m}{ }^{2}\right)$ & $85.3 \pm 32.7(25.7-185.4)$ & $47.0 \pm 22.4(21.64-144.81)$ & $0.030^{*}$ \\
E $(\mathrm{mSv})$ & $0.24 \pm 0.09(0.07-0.52)$ & $0.13 \pm 0.06(0.06-0.41)$ & $<0.001^{*}$ \\
\hline
\end{tabular}

Mean \pm SD is given for all measurements. The range for all results is given between round brackets. Significant differences are indicated by an *
Table 5 Objective and subjective image quality results: SNR (bone and soft tissue) and total score

\begin{tabular}{lcll}
\hline & Group A & Group B & $P$ value \\
\hline Protocol & Standard & Optimized & \\
SNR bone & $\begin{array}{c}76.8 \pm 23.5 \\
(49-150)\end{array}$ & $45.8 \pm 11.9(29-78)<0.001^{*}$ \\
SNR soft tissue & $\begin{array}{c}31.8 \pm 10.47 \\
(16-69)\end{array}$ & $22.4 \pm 6.3(12-40)<0.001^{*}$ \\
Total mean score & $28.1 \pm 2.4(21-30)$ & $27.9 \pm 2.3(22-30)$ & 0.612 \\
\hline
\end{tabular}

Mean \pm SD is given for all measurements. The range for all results is given between round brackets. Significant differences are indicated by an *

We observed a difference in objective image quality between the standard and the optimized protocol. With the optimized protocol signal-to-noise ratio (SNR) for bone and soft tissue reduced from $76.8 \pm 23.5$ (bone) and $31.8 \pm 10.47$ (soft tissue) to $45.8 \pm 11.9$ (bone) and $22.4 \pm 6.3$ (soft tissue), respectively (Table 5).

Figure 4 provides an illustration of images of protocols A and B. Subjective image quality was not observed to be different ( $p$ values for all six evaluated criteria were between 0.320 and 0.837). Table 6 shows the results of the image quality assessment. Interobserver agreement (Table 7) varied from moderate to almost perfect, depending on the criteria that were scored.

\section{Discussion}

Owing to the increased radiation sensitivity of children, the potential risk of multiple radiographic examinations in the management of scoliosis has led to efforts to reduce exposure on the basis of the ALARA principle [4, 6, 7, 10-12]. This includes limiting the number of radiographs [13], development of competing digital technologies and optimizing existing protocols $[7,10,12]$. With this study, an optimization of the existing protocol was performed by adjusting the acquisition parameters together with image quality assessment. This process could be easily performed in a clinical environment. Since the first examination of scoliosis patients requires a high-quality anatomical image, our goal was to optimize our standard protocol without compromising

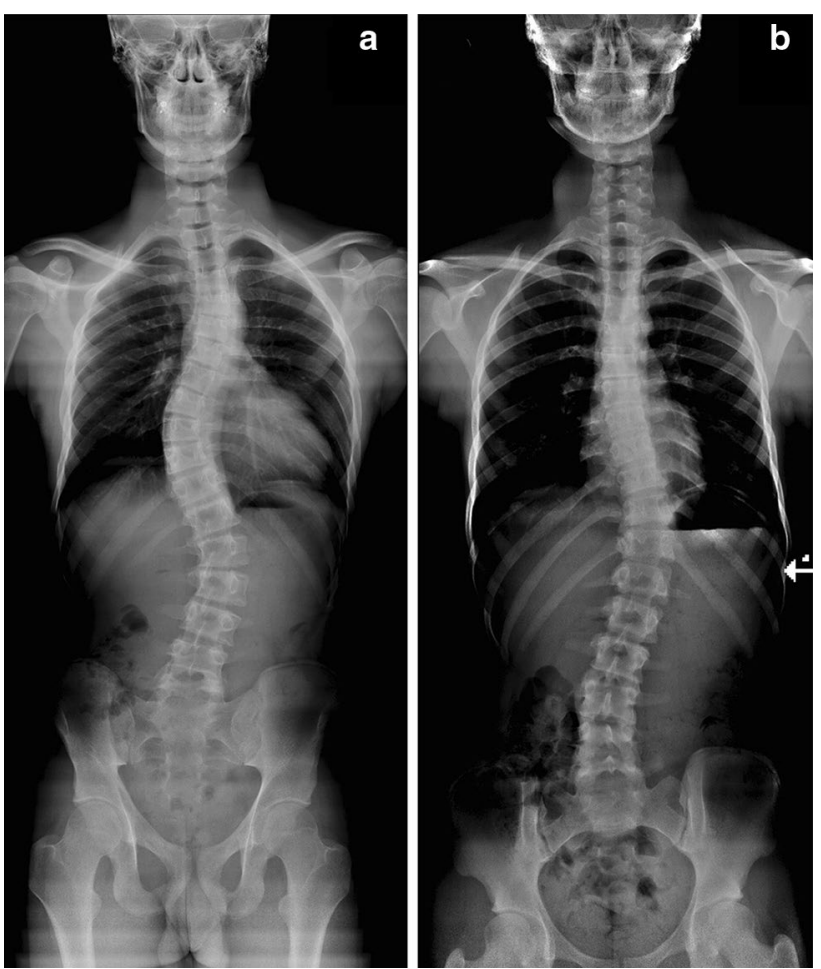

Fig. 4 Whole spine PA radiographs of adolescents with idiopathic scoliosis performed with our standard protocol (a) and optimized protocol (b)

Table 6 Results of subjective image quality assessment, statistically analyzed by the Mann-Whitney $U$ test

\begin{tabular}{lccl}
\hline Criteria & Group A & Group B & $P$ \\
\hline Bone/soft tissue contrast (1-5) & $4.8 \pm 0.5$ & $4.8 \pm 0.4$ & 0.601 \\
Bone sharpness (1-5) & $4.7 \pm 0.5$ & $4.7 \pm 0.4$ & 0.713 \\
Visibility of processus spinosus (1-5) & $4.5 \pm 0.6$ & $4.4 \pm 0.7$ & 0.499 \\
Delignation of the intervertebral spaces & $4.9 \pm 0.3$ & $4.9 \pm 0.3$ & 0.837 \\
$\quad(1-5)$ & & & \\
Assessment of the spinal curve (1-5) & $4.9 \pm 0.3$ & $5 \pm 0$ & 0.320 \\
Assessment of the Risser grade (1-5) & $4.2 \pm 1.5$ & $4.1 \pm 1.5$ & 0.780 \\
\hline
\end{tabular}

Mean \pm SD is given for all measurements 
Table 7 Interobserver agreement for the different criteria and for the overall score

\begin{tabular}{ll}
\hline Criterion & ICC \\
\hline Overall score & $0.958(0.934,0.973)$ \\
Bone/soft tissue contrast & $0.930(0.890,0.955)$ \\
Bone sharpness & $0.560(0.314,0.718)$ \\
Visibility of processus spinosus & $0.883(0.817,0.925)$ \\
Delignation of the intervertebral spaces & $0.786(0.667,0.863)$ \\
Assessment of the spinal curve & $\mathrm{NA}$ \\
Assessment of the Risser grade & $0.919(0.874,0.948)$ \\
\hline
\end{tabular}

The range for all results is given between round brackets

$<0$ poor agreement, $0.0-0.20$ slight agreement, $0.21-0.40$ fair agreement, $0.41-0.60$ moderate agreement, $0.61-0.80$ substantial agreement, $0.81-1$ almost perfect agreement, NA not available due to lack of intrareader variance

image quality, this obviously within the possibilities of our X-ray system.

Our optimization process included a phantom study to understand the impact of technical parameters on dose and image quality. From this phantom study, we identified a protocol which had similar image quality at the lowest radiation dose. We then applied this protocol on a patient study. The dose gains were achieved by adding a $2-\mathrm{mm} \mathrm{Cu}$ filter and lowering relative exposure (protocol 8, Table 3) which resulted in a dose reduction of approximately $70 \%$ in our phantom study and $45 \%$ for our patient study. The tube potential of $84.8 \mathrm{kV}$ remained and is within the limits of $65-90 \mathrm{kV}$ stated by the Commission of the European Communities as examples of good radiographic technique for a full-spine examination in pediatrics $[14,15]$. From the phantom study, we also observed that the lowest radiation dose could be achieved by removing the anti-scatter grid but this at cost of the subjective IQ (protocol 11 Table 3). Although the loss in image quality was minor (mean total score of 29.5 for this protocol in comparison with a score of 30 for protocol A), and would not necessarily affect the correct diagnosis and/or therapeutic decisions, the aim of our study was to preserve our initial image quality. This protocol was therefore, inevitably rejected to use for our patient study but could be considered in studies where only spinal curve measurements are required. Although our lowered dose images result in higher image noise levels, as expressed by a significant lower signal-to-noise ratio (SNR) compared to the standard protocol images, the visual grading of the images indicated that there was no significant difference in score, and thus image quality between both protocols was perceived as similar (Table 5). Interreader agreement was found to be moderate to almost perfect for both groups.

Previous studies already evaluated the effective doses at scoliosis examination [12, 14, 16-19]. The reported effective doses varied between 0.02 and $0.49 \mathrm{mSv}$. One study compared conventional film/grid technique $(\mathrm{F} / \mathrm{G})$ with air-gap technique using computed radiography (CR) [12], two studies $[17,18]$ used an air-gap technique using conventional films and the other three studies performed the scoliosis examinations by digital-pulsed fluoroscopy [14, $16,19]$. In four of these studies, the effective doses were determined based on the Monte Carlo calculation using PCXMC or a similar computer program [12, 14, 16, 18], whereas the estimation in two analyses involved calculating the total energy transfer to the organs, based on air kerma or TLD measurements [17, 19]. Although there is no accepted standard method for the determination of the effective radiation doses of radiological procedures in children, Geijer $\mathrm{H}$. et al. [16] found a remarkably good correlation between the measured values of effective dose and the Monte Carlo calculations which indicates that these calculations are a useful tool in the clinical setting for the evaluation of the effective dose. The lowest doses in these studies $(0.02 \mathrm{mSV})$ were achieved by digital fluoroscopy [17] and were significantly lower than the effective dose from our optimized protocol (Table 2), however, the image quality in this study was described as lower but acceptable for accurate angle measurements, whereas the image quality in our study was preserved (Table 5). Other studies compared radiation dose and image quality between a slot-scanning device (SSD) (EOS imaging) and a digital radiographic system in assessing scoliosis in children [10, 20]. SSD has the possibility to simultaneously acquire two orthogonal images, which facilitates three-dimensional surface reconstruction of vertebrae what makes this system interesting from a clinical perspective. Significant dose savings with an equivalent or superior image quality were reported with the SSD, however, effective doses are rarely determined which makes comparison with our study more difficult. Yvert et al. [10] reported mean DAP values of $39.8 \pm 11.7 \mathrm{cGy} \mathrm{cm}^{2}$ for standing whole-spine frontal radiographs. These values are comparable with the DAP values of our optimized protocol $(47.0 \pm 22.4)$ taking into account the fact that the mean age in this study (11.4 \pm 2.6 years) was somewhat lower compared to our study $(13.4 \pm 1.7$ years). Two studies reported on microdose X-ray (EOS) protocols for patients with adolescent idiopathic scoliosis where the DAP values were between $3.9 \pm 0.7 \mathrm{cGy} \mathrm{cm}^{2}[21]$ and $4.3 \pm 1.3 \mathrm{cGy} \mathrm{cm}^{2}$ [22]. These dose values are significantly lower than our reported doses, however, the image quality assessment mainly concerned static parameters (e.g., Cobb angle measurements, tilting assessment) and technology (e.g., collimation), whereas in our study, we performed a more detailed radiological image quality assessment including the evaluation of bone sharpness and the visibility or delignation of specific anatomical structures, which again, makes a comparison with our study more difficult. A drawback of a SSD system is its higher cost. In a health economics study that compared SSD to 
standard X-ray, McKenna et al. demonstrated that an SSD system can be shown only to be cost-effective if the utilization is about double than standard X-ray [23]. This suggests that an optimized conventional radiographic system can provide a dose- and cost-effective solution for the diagnosis and follow-up of idiopathic scoliosis.

Some limitations of our work have to be considered. Most importantly, these results have only be validated when using the Axiom Luminos dRF from Siemens, and therefore they cannot be directly extrapolated to other types of X-ray systems. Second, this study only included patients without brace who were able to hold a standing position, results could differ for supine or in-brace radiographs.

In summary, with a dose reduction of $45 \%$, image quality remained similar in terms of bone/soft tissue contrast, bone sharpness, visibility of processus spinosus, delignation of the intervertebral spaces, assessment of the Risser grade and assessment of the spinal curve. We conclude that, in the diagnosis and follow-up of idiopathic scoliosis, a dedicated lowered dose full-spine radiograph protocol can replace the standard protocol with similar image quality.

\section{Compliance with ethical standards}

Conflict of interest None of the authors has any potential conflict of interest.

Open Access This article is distributed under the terms of the Creative Commons Attribution 4.0 International License (http://creativeco mmons.org/licenses/by/4.0/), which permits unrestricted use, distribution, and reproduction in any medium, provided you give appropriate credit to the original author(s) and the source, provide a link to the Creative Commons license, and indicate if changes were made.

\section{References}

1. Stirling AJ, Howel D, Millner PA et al (1996) Late-onset idiopathic scoliosis in children six to fourteen years old. A cross-sectional prevalence study. J Bone Joint Surg Am 78(9):1330-1336

2. Presciutti SM, Karukanda T, Lee M (2014) Management decisions for adolescent idiopathic scoliosis significantly affect patient radiation exposure. Spine J 14(9):1984-1990

3. Mgaadi M, Ben Omrane L, Hammou A (2012) Effective dose for scoliosis patients undergoing full spine radiography. Radiat Prot Dosimetry 149(3):297-303

4. Pace N, Leonardo R, Negrini S (2013) A comparison approach to explain risks related to X-ray imaging for scoliosis, 2012 SOSORT award. Scoliosis 8(1):11

5. Amzallag-Bellenger E, Uyttenhove E, Nectoux E et al (2014) Idiopathic scoliosis in children and adolescents: assessment with a biplanar X-ray device. Insights into imaging [serial online] 5:571-583

6. Frush DP, Donelly LF, Rosen NS (2011) Radiation risk from medical imaging: a special need to focus on children. In: Evidencebased imaging. Improving the quality of imaging in patient care. Springer, New York, pp 27-41

7. Law M, Ma WK, Lau D et al (2016) Cumulative radiation exposure and associated cancer risk estimates for scoliosis patients: impact of repetitive full spine radiography. Eur J Radiol $85: 625-628$

8. Hohenforst-Schmidt W, Banckwitz R, Zarogoulidis P et al (2014) Radiation exposure of patients by cone beam CT during endobronchial navigation : a phantom study. J Cancer 5(3):192-202

9. Båth M (2010) Evaluating imaging systems: practical applications. Radiat Prot Dosimetry 139:26-36

10. Yvert M, Diallo A, Bessou P et al (2015) Radiography of scoliosis: comparative dose levels and image quality between a dynamic flat-panel detector and a slot-scanning device (EOS system). Diagn Interv Imaging 96(11):1177-1188

11. ICRP (2007) The 2007 Recommendations of the International Commission on Radiological Protection. ICRP Publication 103. Ann. ICRP 37 (2-4)

12. Hansen J, Jurik AG, Fiirgaard B et al (2003) Optimisation of scoliosis examinations in children. Pediatr Radiol 33(11):752-765

13. Andersen P Jr, Andersen P, van der Kooy P (1982) Dose reduction in radiography of the spine in scoliosis. Acta Radiol Diagn 23:251-253

14. Geijer H, Verdonck B, Beckman KW et al (2003) Digital radiography of scoliosis with a scanning method: radiation dose optimization. Eur Radiol 13(3):543-551

15. Commission of the European Communities (1996) European guidelines on quality criteria for diagnostic radiographic images in pediatrics. Office for official publications of the European Communities, EUR 16261

16. Geijer H, Beckman KW, Jonsson B et al (2001) Digital radiography of scoliosis with a scanning method: initial evaluation. Radiol 218:402-410

17. Hallen S, Martling K, Mattsson S (1992) Dosimetry at X ray examinations of scoliosis. Radiat Prot Dosimetry 43:49-54

18. Almen AJ, Mattsson S (1996) Dose distribution at radiographic examination of the spine in pediatric radiology. Spine 21:750-756

19. Chamberlain C, Huda W, Hojnowski L et al (2000) Radiation doses to patients undergoing scoliosis radiography. Br J Radiography 73:847-853

20. Deschênes S, Charron G, Beaudoin G et al (2010) diagnostic imaging of spinal deformities: reducing patients dose with a new slot-scanning X-ray imager. Spine 35(9):989-994

21. Hui S, Pialasse JP, Wong J et al (2016) Radiation dose of digital radiography (DR) versus micro-dose X-ray (EOS) on patients with adolescent idiopathic scolios: 2016 SOSORT_IRSSD "John Sevastic Award” winner in imaging research. Scoliosis Spinal Disord $11: 46$

22. Ilharreborde B, Ferrero C, Alison M et al (2016) EOS microdose protocol for the radiological follow-up of adolescent Idiopathic scoliosis. Eur Spine J 25:526-531

23. McKenna C, Wade R, Faria R et al (2012) EOS 2D/3D X-ray system: a systematic review and economic evaluation. Health Technol Assess 16(14):1 